\title{
Study of the Mechanical and Morphology Properties of Recycled HDPE Composite Using Rice Husk Filler
}

\author{
Jia Ying Tong, ${ }^{1}$ Nishata Royan Rajendran Royan, ${ }^{1}$ Yong Chuen $\mathbf{~ g g},{ }^{1}$ \\ Mohd Hafizuddin Ab Ghani, ${ }^{2}$ and Sahrim Ahmad ${ }^{2}$ \\ ${ }^{1}$ Faculty of Applied Science, UCSI University, 56000 Cheras, Kuala Lumpur, Malaysia \\ ${ }^{2}$ Faculty of Science and Technology, Universiti Kebangsaan Malaysia, 43600 Bangi, Selangor, Malaysia \\ Correspondence should be addressed to Nishata Royan Rajendran Royan; ry_nish@yahoo.com
}

Received 22 May 2013; Revised 26 December 2013; Accepted 27 December 2013; Published 18 February 2014

Academic Editor: Luigi Nicolais

Copyright (C) 2014 Jia Ying Tong et al. This is an open access article distributed under the Creative Commons Attribution License, which permits unrestricted use, distribution, and reproduction in any medium, provided the original work is properly cited.

\begin{abstract}
WPCs are being used in a large number of applications in the automotive, construction, electronic, and aerospace industries. There are an increasing number of research studies and developments in WPC technology involving rice husk as fillers. This study investigated the effects of different compositions of rice husk $(\mathrm{RH})$ filler on the mechanical and morphological properties of recycled HDPE (rHDPE) composite. The composites were prepared with five different loading contents of RH fibers (0, 10, 20, 30, and $40 \mathrm{wt} \%)$ using the twin screw extrusion method. Maleic acid polyethylene (MAPE) was added as a coupling agent. Results showed that tensile and flexural properties improved with increasing RH loading. However, the impact strength of the composites decreased as the RH loading increased. SEM micrographs revealed good interfacial bonding between the fiber and polymer matrix.
\end{abstract}

\section{Introduction}

Wood-plastic composites (WPCs) were born as a modern concept in Italy in the 1970s. In the early 1990s, WPCs were popularized in North America and had spread to Singapore, India, Malaysia, Japan, and China by the start of 21st century [1]. WPCs are being used in a large number of applications in automotive, construction, electronic, and aerospace industries. The major application of green WPCs in the market is to replace fiberglass and steels. There are an increasing number of research studies and developments in the WPC technology recently.

Over the last few years, there are many researches on the natural fibers as loading constituents in composite materials. The biodegradability, low costs, environmental friendliness, low density, non-hazardous, nonabrasive nature and wide variety types of natural fibers are important factors for their acceptance in large volume markets [2]. Rice husk (RH) is one of the waste materials in the rice growing regions such as Malaysia, Thailand, Vietnam, India, Korea, and other Asia countries [3]. Generally, rice husk contains 35\% cellulose, $25 \%$ hemicellulose, $20 \%$ lignin, and $17 \%$ ash (94\% silica) by weight depending on the geographic location [4].
Rice husk mixed with resin polymers producing composite materials has gained public attention. Many researches are currently conducted on the studies of rice husk ash and rice husk reinforced thermoplastic composites. Most of the studies conducted are focused on material characterization and mechanical properties of the rice husk reinforced composites. Naurah [5] studied the effect of the presence of rice husk composed of $10 \%$ to $40 \%$ of total weight in high density polyethylene (HDPE) and the result has shown that $30 \%$ rice husk provided the optimum mechanical properties. Composites containing more than $50 \%$ rice husk were unable to be produced as they cause blockages resulting in damage to the extruder. Therefore, composites contained more than $50 \%$ of rice husk using traditional extrusion are strongly discouraged.

According to Lu et al. [6], a coupling agent accounts for only $1-3 \%$ of the total weight of composites. Maleic anhydride is the most commonly used among different coupling agents. In general, reports have proven maleic anhydride grafted matrices used as compatibilizer able to improve the tensile strength and elongation at break [7]. Such improvement is due to the formation of ester bonds 
between anhydride groups of coupling agents entered into an esterification reaction with the surface hydroxyl groups of wood fibers $[8,9]$. The coupling agent is chemically bonded with hydrophilic fiber and blended by wetting in the polymer chain [9]. Adhikary et al. [10] observed that the tensile strength of the composite and its increase in stiffness was due to the addition of coupling agent (maleated polypropylene). Fuqua and Ulven [11] studied the different maleated polypropylene (MAPP) loading ( 0,5 , and $10 \mathrm{wt} \%)$ effects on tensile properties of corn chaff fiber reinforced polypropylene composites. It was found that $5 \mathrm{wt} \% \mathrm{MAPP}$ yielded the optimum value for the composites in terms of tensile strength and modulus. The reduced strength with high MAPP loading was caused by the interaction between MAPP and the fiber/matrix system.

The main objective of this study was to prepare recycled HDPE composites reinforced with rice husk filler with addition of $4 \mathrm{wt} \%$ coupling agent. The effects of different composition of rice husk filler on its mechanical and morphological properties of the composites are reported in this study.

\section{Experimental Procedures}

Recycled HDPE is used as a matrix in the composites whilst the rice husk (RH) used as filler was supplied by RePlas Sdn Bhd. The density of rHDPE is $923 \mathrm{~kg} / \mathrm{m}^{3}$. The melt flow index (MFI) of rHDPE is $0.072 \mathrm{~g} / 10 \mathrm{~min}$ at $190^{\circ} \mathrm{C}$. Maleic anhydride grafted polyethylene (MAPE) is used as coupling agent. RH fibers are dried overnight at $90^{\circ} \mathrm{C}$ in a vacuum oven to reduce its moisture content. The $\mathrm{RH}$ fiber was used without any subsequent treatment.

In the first stage, a laboratory scale counterrotating twin screw extruder (Thermo Prism TSE 16PC) was employed for compounding RH fibers and rHDPE with the coupling agent. Five different fiber loadings $(0,10,20,30$, and $40 \mathrm{wt} \%)$ were used. The barrel temperatures of the four zones were $180^{\circ} \mathrm{C}$, $190^{\circ} \mathrm{C}, 200^{\circ} \mathrm{C}$, and $190^{\circ} \mathrm{C}$. The screw speed was $30 \mathrm{rpm}$. The extrudates were collected, cooled, and granulated into pellets. The pellets collected were then pelletized using a crusher machine, so that the pellets can fit into improve mould. In the second stage, hot and cold press process (LP50, LABTECH Engineering Company LTD) was used to make the specimen panels for testing. Pellets were put into mould $14 \mathrm{~mm} \times 14 \mathrm{~mm}$ $\times 3 \mathrm{~mm}$ thick. The temperature of the hot press was set at $190^{\circ} \mathrm{C}$ for both upper heater and lower heater. The period of preheating, venting, and full pressing was set to 3,2 , and 5 minutes, respectively. The cold press was set to 5 minutes to cool down the specimens. The pressure used to press the samples was set at $6894.75 \mathrm{kPa}$. These parameter controls were adapted from Ab Ghani and Ahmad [12].

Prepared specimens were characterized by tensile, flexural, and notched impact tests. Tensile tests were conducted according to ASTM D 638-03 using Materials Testing Machine, model: M350-10CT with the speed of $5 \mathrm{~mm} / \mathrm{min}$. Flexural test was performed according to ASTM D 790-03 using Materials Testing Machine, model: M350-10CT. Three point flexural test with the crosshead speed of $5 \mathrm{~mm} / \mathrm{min}$ was used in this experiment. Ray-Ran Universal Pendulum

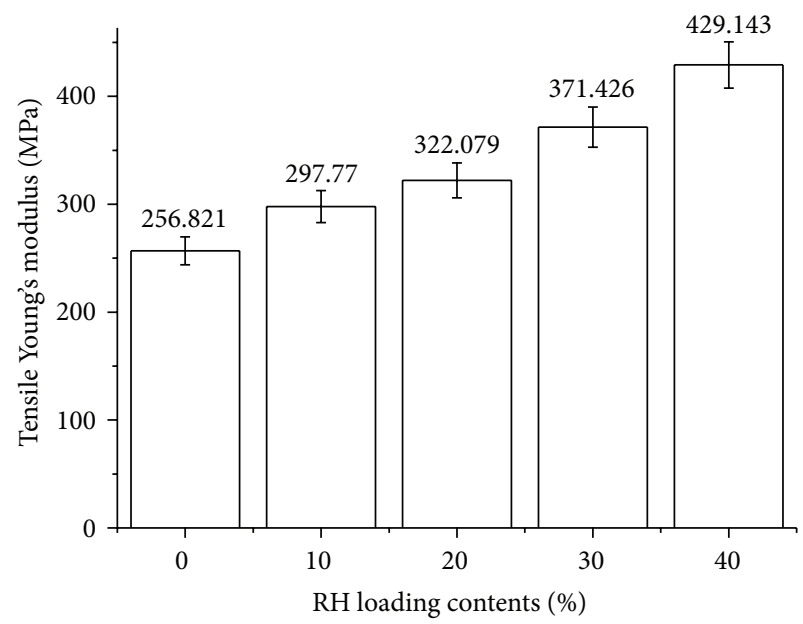

FIgURE 1: Tensile Young's modulus of composites with different loading of rice husk fibers.

Impact System was used to determine the impact strength of the specimens. The specimens were cut according to ASTM D 256-05 specification. The velocity of the pendulum was $3.46 \mathrm{~m} / \mathrm{s}$ and the weight used was $0.452 \mathrm{~kg}$. The fracture surfaces of the specimens that contained $0 \%, 20 \%$, and $40 \%$ rice husk fibers were characterized with scanning electron microscope (SEM). The SEM was operated at an accelerating voltage of $15 \mathrm{kV}$ after being sputter coated with gold.

\section{Results and Discussion}

3.1. Tensile Test. Tensile test was performed to measure the response of a material to slowly applied uniaxial force [13]. Figure 1 illustrates the result of tensile test. Tensile test was performed according to ASTM D 638-03 at room condition using a testometric universal testing machine with a loading rate of $1 \mathrm{~mm} / \mathrm{min}$ for Young's modulus determination. Results obtained from tensile modulus determinations indicated that an increase rice husk loading has the tendency to increase the composite stiffness as showed in Figure 1. Pure HDPE composite has the lowest tensile modulus compared with the reinforced HDPE with rice husk composites. Increasing the rice husk fiber in the composite would increase the tensile modulus of the composite. Forty percent of rice husk loading in the HDPE composite showed the highest tensile modulus which is $429.143 \mathrm{MPa}$.

Some studies were experimented on sisal fiber-polypropylene composites [14], flax fiber-polypropylene composites, and flax fiber-polylactic acid composites [15]. The authors found that the incorporation of natural fiber into plastic increased the tensile strength in comparison with the unreinforced plastic matrix. Luz et al. [16] indicated that natural lignocellulosic fillers have a higher elastic modulus than the polymer matrix. This is because the rigidity of its composites tends to increase strongly with the addition of these fillers.

Young's modulus shows an increase with the natural filler loading that was in accordance with other reported works 


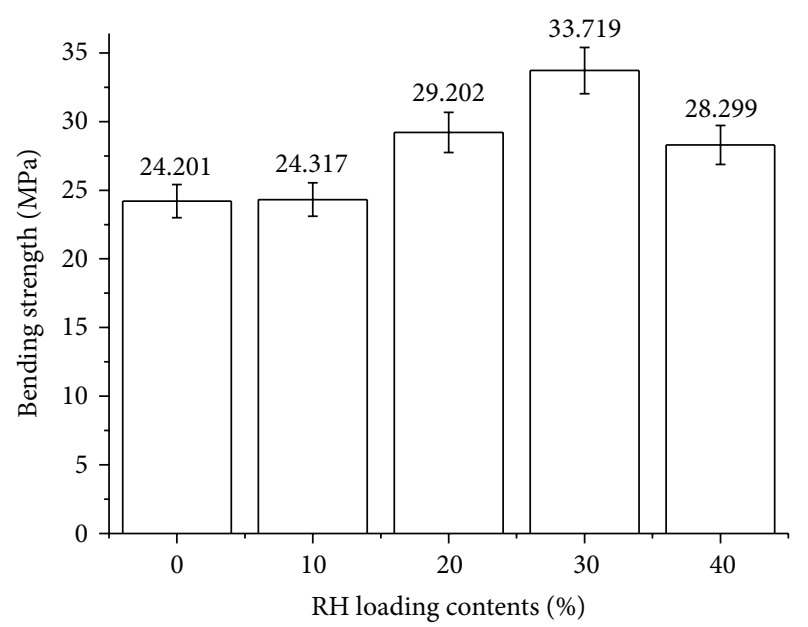

FIGURE 2: Bending strength of composites with different loading of rice husk fibers.

[17-20]. According to Rahman et al. [21], partially separated microspaces are created during tensile loading. These would obstruct the stress propagation between the filler and the matrix. Thus, as the filler loading increases, the degree of obstruction increases which in turn increases the stiffness.

Yang et al. [18] mentioned in his research that tensile strength improved with an increase in the compatibilizing agent content in his research. The improved interfacial bonding between the filler and matrix polymer resulted in good stress propagation and improved the tensile strength. Such improvement is due to the formation of ester bonds between anhydride groups of coupling agents entered into an esterification reaction with the surface hydroxyl groups of wood fibers [9]. This statement was supported by Ismail et al. [22] and Rana et al. [19]. The test temperature was maintained at room temperature to avoid the temperature affecting the test result of tensile properties. As the test temperature increases, both tensile strength and modulus decreased while the composite gradually exhibits plastic deformation [22].

$\mathrm{RH}$ acts as reinforcement filler in rHDPE that showed good tensile properties. In order to the interfacial bonding between RH and rHDPE and enhance the tensile properties of the composites, novel processing techniques and chemical and physical modification methods can be carried out in further research. According to Ku et al. [23], it is obviously shown that the strength and stiffness of the natural fiber polymer composites are strongly dependent on fiber loading. The tensile strength and modulus increase when the fiber loading ratios increase up to a certain amount. If the fiber loading ratio increases below optimum value, load is distributed to more fibers which are well bonded with matrix and hence resulting better tensile properties. Further increase in the amount of fiber loading resulted in decreased tensile strength.

3.2. Flexural Test. Flexural test was performed according to ASTM D 790-03 at room condition using testometric universal machine for modulus of rupture (MOR) determination and modulus of elasticity (MOE) investigation. Figures 2, 3,



FIgURE 3: Bending modulus of composites with different loading of rice husk fibers.

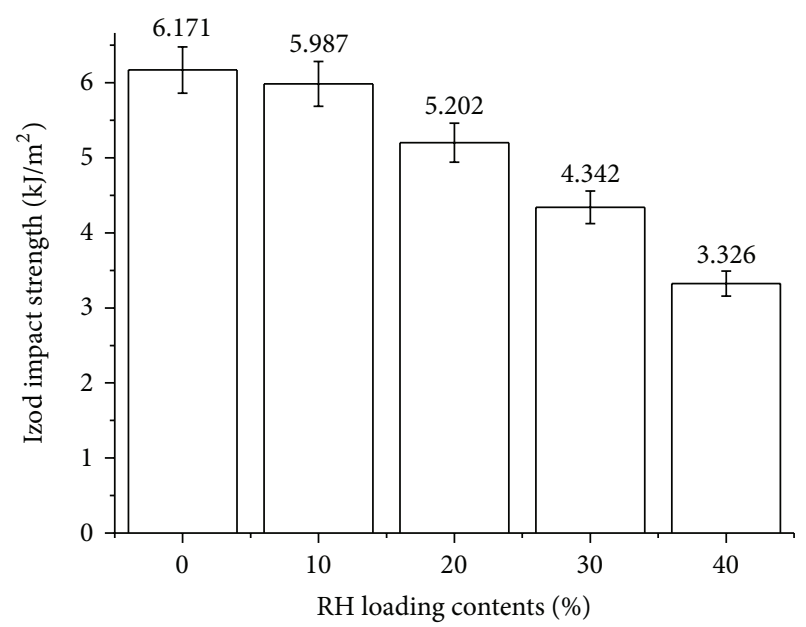

FIGURE 4: Impact strength of composites with different loading of rice husk fibers.

and 4 illustrate the results of flexural test. Figure 2 shows the value of transverse rupture strength (MOR) against rice husk loading contents. From the graph, the modulus of rupture increased as increasing the rice husk fiber loading. The modulus of rupture decreased at $40 \%$ of rice husk loading. The bending strength increased as the rice husk loading increased. As can be seen in Figure 3, the bending strength started to decrease at $40 \%$ of rice husk loading. Figure 4 shows the value of bending modulus (MOE) against rice husk loading contents. The trend increased as the rice husk loading increased.

According to Panthapulakkal and Sain [24], the modulus did not change with the incorporation of compatibilizing agents as the modulus is mainly dependent on the filler content in the composites. Ghofrani et al. [25] mentioned that $\mathrm{MOE}$ reduced considerably by increasing the content of HDPE and that result is supported by previous researches. Increasing HDPE significantly improved the bending strength and MOE. Referring to the research of 


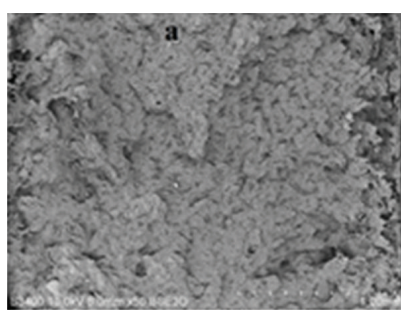

(a)

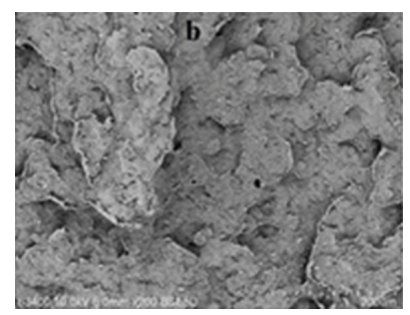

(b)

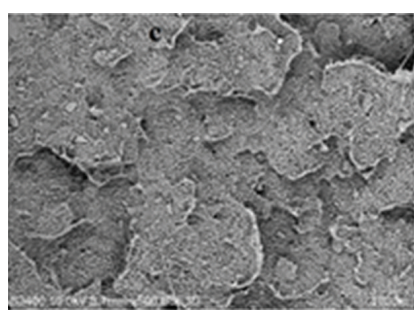

(c)

FIGURE 5: : SEM micrograph of the surface on 0\% RH loading contents composites: (a) 50x magnification, (b) 200x magnification, and (c) 500x magnification.

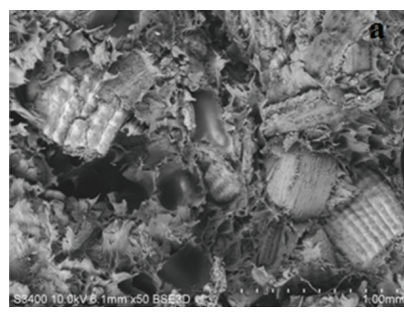

(a)



(b)

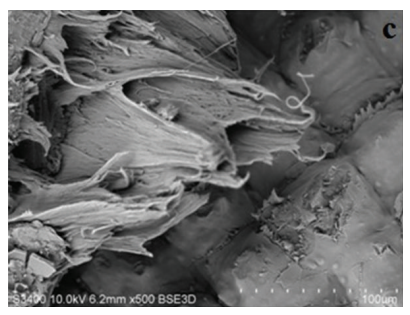

(c)

FIGURE 6: SEM micrograph of the surface on $20 \%$ RH loading contents composites: (a) 50x magnification, (b) 200x magnification, and (c) 500x magnification.

Rahman et al. [21], the bending strength increased with filler loading up to $35 \%$, but the strength was decreased at $40 \%$. Besides, the bending modulus of RH reinforced composites increased with higher filler loading. Similar results with different reinforced materials were reported in previous researches $[19,20,26]$. Therefore, further increase of the filler loading in this research is not highly recommended.

3.3. Impact Test. Impact test is to measure the ability of a material to absorb the sudden application of a load with breaking [12]. The impact tests were carried out according to ASTM D 256-05 at room condition using a Universal Pendulum Impact System (Ray-Ran, UK). Figure 5 shows the results of the impact tests. The graph Figure 5 indicates that the impact strength of the composites decreases as the rice husk loading increases. The pure HDPE composite has the highest impact strength compared to the rice husk reinforced composites.

The interaction between the neighboring fibers appears to block matrix flow which causes the embrittlement of the polymer matrix. This behavior can decrease the impact strength of the composites. The reduction of impact energy during crack formation and failure has been noted in a previous study [27]. Ashori and Nourbakhsh [28] also mentioned that the reduced flexibility of polymer matrix is another reason for decreasing impact strengths.

According to Yang et al. [17], the notched samples exhibited lower impact strength compared to the unnotched samples. The notched tip is the stress concentrating point when impact occurs. With the addition of coupling agent, the interfacial bonding between the filler and matrix polymer was highly improved. Initially, the interface between $\mathrm{RH}$ fibers and the polymer matrix is weak due to the incompatibility between the hydrophobic wood fibers and the hydrophilic polymer matrix. Therefore, when treated with coupling agent, the chemical composition of coupling agents allows them to react with the fiber surface by forming a bridge of chemical bonds. Thus, when the impact occurred, the crack was not initiated at the interface but at the filler itself. The filler which is more brittle, compared to matrix polymer, makes the impact strength of the composites decrease. Notched impact behavior is controlled to a greater extent by factors that affect the propagation of fractures initiated at the predominating stress concentration at notched tip [22].

3.4. Morphology. The fracture surfaces of the tensile test specimens were examined using a scanning electron microscope (SEM). The SEM micrograph image of $0 \mathrm{wt} \%$ rice husk fiber is shown in Figures 5(a), 5(b), and 5(c). There are no clear gaps in between the pure rHDPE particles. This proves that the rHDPE had been mixed well during the extrusion process. The optimum parameter was used in order to produce a good composite while providing high mixing efficiency. Figures 6(a), 6(b), and 6(c) show the SEM micrograph image of $20 \mathrm{wt} \%$ rice husk fibers. The SEM image showed that there were no clear gaps between $\mathrm{RH}$ fibers and recycled HDPE matrix, indicating good interface bonding. SEM images of the rice husk-recycled HDPE composites at filler loading of $40 \mathrm{wt} \%$ are shown in Figures $7(\mathrm{a}), 7(\mathrm{~b})$, and $7(\mathrm{c})$ in 50x, 200x, and 500x magnification, respectively. The fibers were perfectly attached to the matrix, and it is also evident that the fibers were strongly imbedded in the matrix. This 


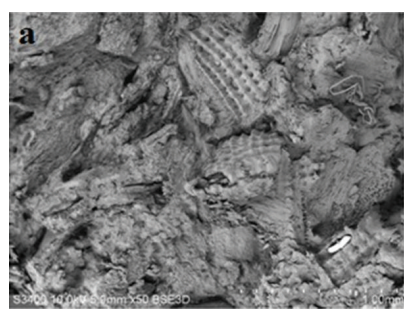

(a)

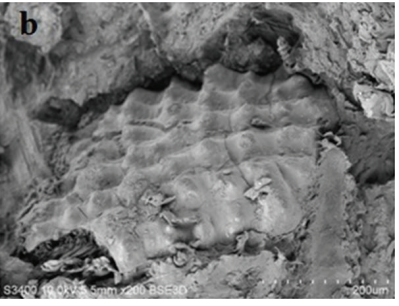

(b)

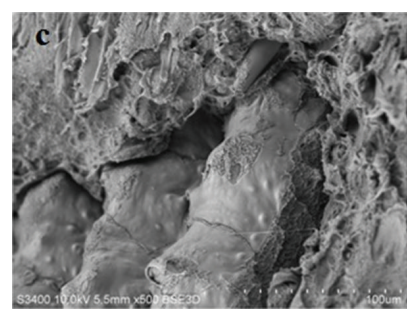

(c)

FIGURE 7: SEM micrograph of the surface on 40\% RH loading contents composites: (a) 50x magnification, (b) 200x magnification, and (c) 500x magnification.

indicates the efficiency of mixing which was attributed to a good interfacial interaction between RH fibers and matrix. Interfacial bonding was improved due to the esterification mechanism and the fracture occurred at the fiber itself. This illustrates that stress is well propagated between the fibers and the matrix polymer resulting in enhanced tensile modulus in response to stress [10].

\section{Conclusion}

Based on the results and discussion of this study, increasing the $\mathrm{RH}$ fiber in the composite would increase the tensile properties and flexural properties of the composite. The impact strength of the composites decreases as the $\mathrm{RH}$ loading increased. The addition of coupling agent used in this research improved the interfacial bonding between the filler and the matrix polymer. Thus, the crack was not initiated at the interface but at the filler itself when the impact occurred. The filler is more brittle compared to the matrix. Therefore, this causes the impact strength in the composite decrease. The SEM image showed that there were no clear gaps between RH fibers and recycled HDPE matrix, indicating good interface bonding. The fibers were perfectly attached to matrix and it is also evident that the fibers were strongly imbedded in the matrix. This indicates that the efficiency of the mixing which was attributed to a good interfacial interaction between $\mathrm{RH}$ fibers and matrix. These results met the objectives of this study. Further research is suggested to functionalize the RH fillers chemically to enhance the interfacial bonding between the fiber and polymer matrix.

\section{Conflict of Interests}

The authors declare that there is no conflict of interests regarding the publication of this paper.

\section{References}

[1] G. Pritchard, “Two technologies merge: wood plastic composites," Plastics, Additives and Compounding, vol. 6, no. 4, pp. 1821, 2004.

[2] A. Ashori, "Wood-plastic composites as promising greencomposites for automotive industries!" Bioresource Technology, vol. 99, no. 11, pp. 4661-4667, 2008.
[3] R. Kishore, V. Bhikshma, and P. Jeevana Prakash, "Study on strength characteristics of high strength Rice Husk Ash concrete," in Proceedings of the 12th East Asia-Pacific Conference on Structural Engineering and Construction (EASEC '12), pp. 2666-2672, Elsevier Ltd, Hong-Kong, January 2011.

[4] H. G. B. Premalal, H. Ismail, and A. Baharin, "Comparison of the mechanical properties of rice husk powder filled polypropylene composites with talc filled polypropylene composites," Polymer Testing, vol. 21, no. 7, pp. 833-839, 2002.

[5] M. I. Naurah, Injection Moulding Parameters and Performance of Rice Husk-High Density Polyethylene Composites, University Teknologi Malaysia, 2006.

[6] J. Z. Lu, Q. Wu, and H. S. McNabb Jr., "Chemical coupling in wood fiber and polymer composites: a review of coupling agents and treatments," Wood and Fiber Science, vol. 32, no. 1, pp. 88$104,2000$.

[7] B. S. Panigrahy, A. Rana, P. Chang, and S. Panigrahi, "Overview of Flax Fiber reinforced thermoplastic composites," Canadian Biosystems Engineering Journal, vol. 06, no. 165, pp. 1-12, 2006.

[8] K. B. Adhikary, Development of Wood Flour-Recycled Polymer Composite Panels as Building Materials, University of Canterbury, 2008.

[9] H.-S. Yang, H.-J. Kim, H.-J. Park, B.-J. Lee, and T.-S. Hwang, "Effect of compatibilizing agents on rice-husk flour reinforced polypropylene composites," Composite Structures, vol. 77, no. 1, pp. 45-55, 2007.

[10] K. B. Adhikary, S. Pang, and M. P. Staiger, "Dimensional stability and mechanical behaviour of wood-plastic composites based on recycled and virgin high-density polyethylene (HDPE)," Composites B, vol. 39, no. 5, pp. 807-815, 2008.

[11] M. A. Fuqua and C. A. Ulven, "Characterization of polypropylene/corn fiber composites with maleic anhydride grafted polypropylene," Journal of Biobased Materials and Bioenergy, vol. 2, no. 3, pp. 258-263, 2008.

[12] S. Ahmad and M. H. Ab Ghani, "The comparison of water absorption analysis between counterrotating and corotating twin-screw extruders with different antioxidants content in wood plastic composites," Advances in Materials Science and Engineering, vol. 2011, Article ID 406284, 4 pages, 2011.

[13] D. R. Askeland and P. P. Phule, The Science and Engineering of Materials, 5th edition, 2006.

[14] K. L. Fung, X. S. Xing, R. K. Y. Li, S. C. Tjong, and Y.-W. Mai, "An investigation on the processing of sisal fibre reinforced polypropylene composites," Composites Science and Technology, vol. 63, no. 9, pp. 1255-1258, 2003. 
[15] K. Oksman, M. Skrifvars, and J.-F. Selin, "Natural fibres as reinforcement in polylactic acid (PLA) composites," Composites Science and Technology, vol. 63, no. 9, pp. 1317-1324, 2003.

[16] S. M. Luz, J. Del Tio, G. J. M. Rocha, A. R. Gonçalves, and A. P. Del'Arco Jr., "Cellulose and cellulignin from sugarcane bagasse reinforced polypropylene composites: effect of acetylation on mechanical and thermal properties," Composites A, vol. 39, no. 9, pp. 1362-1369, 2008.

[17] M. M. Thwe and K. Liao, "Effects of environmental aging on the mechanical properties of bamboo-glass fiber reinforced polymer matrix hybrid composites," Composites A, vol. 33, no. 1, pp. 43-52, 2002.

[18] H.-S. Yang, H.-J. Kim, J. Son, H.-J. Park, B.-J. Lee, and T.S. Hwang, "Rice-husk flour filled polypropylene composites; mechanical and morphological study," Composite Structures, vol. 63, no. 3-4, pp. 305-312, 2004.

[19] A. K. Rana, A. Mandal, and S. Bandyopadhyay, "Short jute fiber reinforced polypropylene composites: effect of compatibiliser, impact modifier and fiber loading," Composites Science and Technology, vol. 63, no. 6, pp. 801-806, 2003.

[20] S. Joseph, M. S. Sreekala, Z. Oommen, P. Koshy, and S. Thomas, "A comparison of the mechanical properties of phenol formaldehyde composites reinforced with banana fibres and glass fibres," Composites Science and Technology, vol. 62, no. 14, pp. 1857-1868, 2002.

[21] M. R. Rahman, M. N. Islam, M. M. Huque, S. Hamdan, and A. S. Ahmed, "Effect of chemical treatment on rice husk (RH) reinforced polyethylene (PE) composites," BioResources, vol. 5, no. 2, pp. 854-869, 2010.

[22] H. Ismail, M. R. Edyham, and B. Wirjosentono, "Bamboo fibre filled natural rubber composites: the effects of filler loading and bonding agent," Polymer Testing, vol. 21, no. 2, pp. 139-144, 2002.

[23] H. Ku, H. Wang, N. Pattarachaiyakoop, and M. Trada, "A review on the tensile properties of natural fiber reinforced polymer composites," Composites B, vol. 42, no. 4, pp. 856-873, 2011.

[24] S. Panthapulakkal and M. Sain, "Agro-residue reinforced highdensity polyethylene composites: fiber characterization and analysis of composite properties," Composites A, vol. 38, no. 6 , pp. 1445-1454, 2007.

[25] M. Ghofrani, S. Pishan, M. R. Mohammadi, and H. Omidi, "A study on rice-husk/recycled high density polyethylene composites: their physical and mechanical properties," Environmental Science, vol. 9, no. 1, pp. 99-112, 2012.

[26] J.-C. Lin, L. C. Chang, M. H. Nien, and H. L. Ho, "Mechanical behavior of various nanoparticle filled composites at lowvelocity impact," Composite Structures, vol. 74, no. 1, pp. 30-36, 2006.

[27] Y. Cui, S. Lee, B. Noruziaan, M. Cheung, and J. Tao, "Fabrication and interfacial modification of wood/recycled plastic composite materials," Composites A, vol. 39, no. 4, pp. 655-661, 2008.

[28] A. Ashori and A. Nourbakhsh, "Mechanical behavior of agro-residue-reinforced polypropylene composites," Journal of Applied Polymer Science, vol. 111, no. 5, pp. 2616-2620, 2009. 



Submit your manuscripts at http://www.hindawi.com
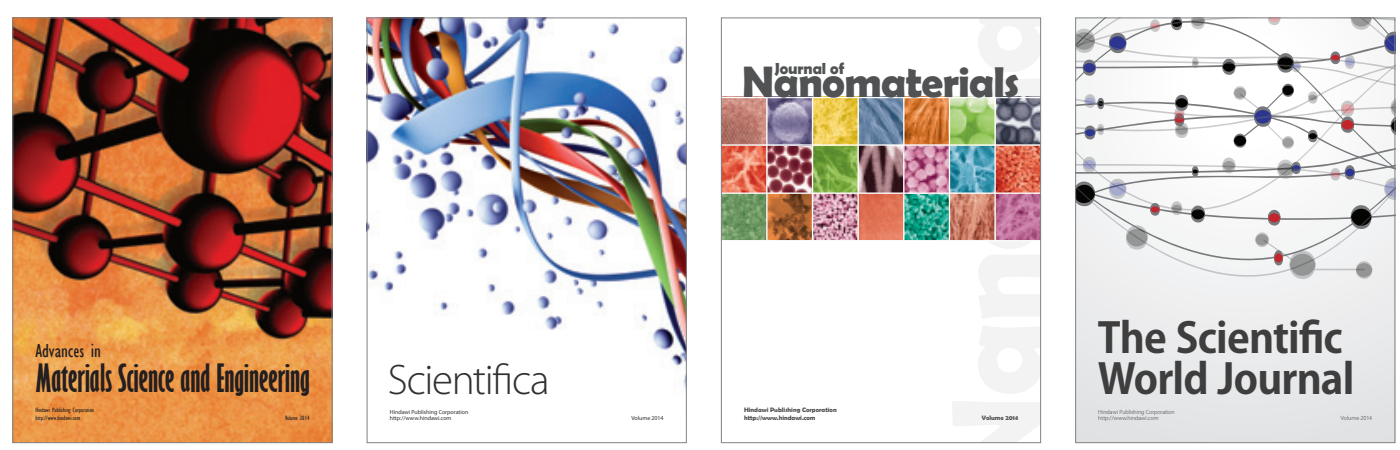

\section{The Scientific World Journal}
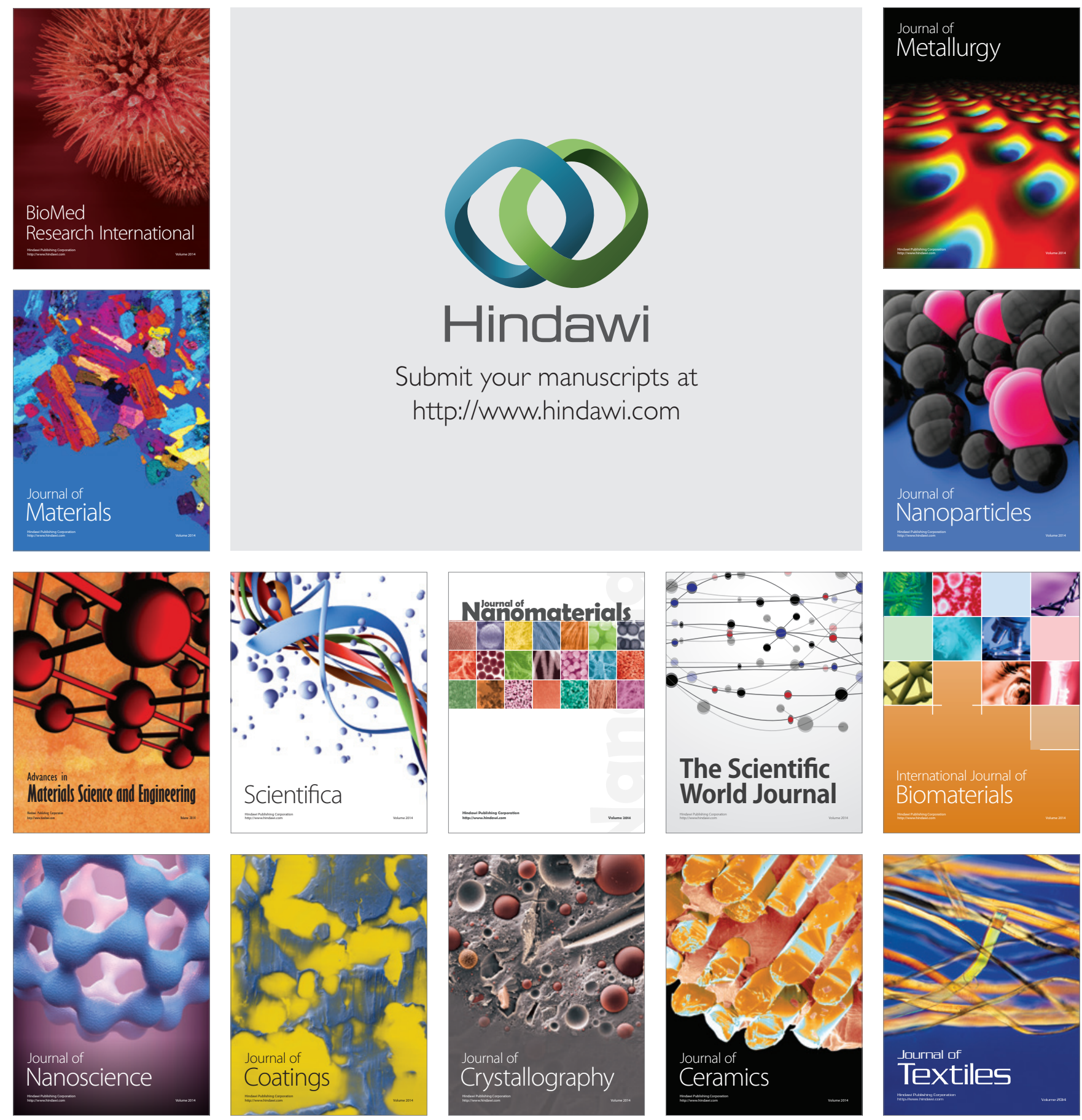